\title{
World economic aid
}

Economic aid² given by non-communist developed countries Average per year (1973-75)

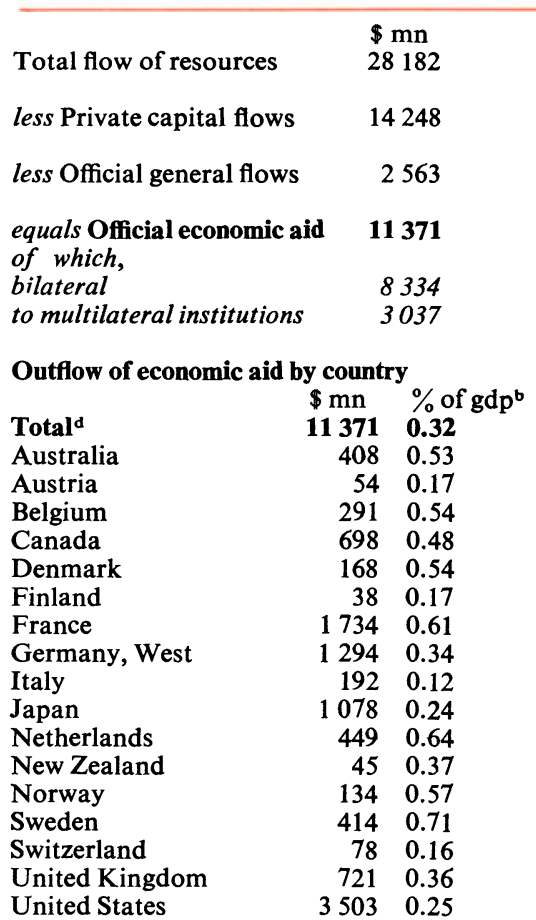

Inflow of economic aid by region and country

\begin{tabular}{|c|c|c|}
\hline Total $^{d}$ & $\begin{array}{l}\$ \mathrm{mn} \\
10647^{\mathrm{c}}\end{array}$ & $\underset{5}{\$ \text { per person }}$ \\
\hline Africa ${ }^{\mathrm{d}}$ & 3028 & 8 \\
\hline Algeria & 120 & 7 \\
\hline Benin & 37 & 12 \\
\hline Botswana & 40 & 61 \\
\hline Burundi & 35 & 10 \\
\hline Cameroon & 76 & 12 \\
\hline Cape Verde & 1 & 4 \\
\hline Central African Empire & 38 & 22 \\
\hline Chad & 55 & 14 \\
\hline Comoros & 22 & 75 \\
\hline Congo & 40 & 29 \\
\hline Djibouti & 28 & 274 \\
\hline Egypt & 188 & 5 \\
\hline Ethiopia & 103 & 4 \\
\hline Gabon & 33 & 63 \\
\hline Gambia & 8 & 13 \\
\hline Ghana & 67 & 7 \\
\hline Guinea & 10 & 2 \\
\hline Guinea-Bissau & 4 & 9 \\
\hline Ivory Coast & 81 & 17 \\
\hline Kenya & 113 & 9 \\
\hline Lesotho & 20 & 20 \\
\hline Liberia & 15 & 9 \\
\hline Libya & 10 & 4 \\
\hline Madagascar & 67 & 9 \\
\hline Malawi & 45 & 9 \\
\hline Mali & 93 & 17 \\
\hline Mauritania & 38 & 30 \\
\hline Mauritius & 21 & 25 \\
\hline Morocco & 128 & 8 \\
\hline Mozambique & 4 & 1 \\
\hline Niger & 99 & 22 \\
\hline Nigeria & 89 & 1 \\
\hline Reunion & 217 & 442 \\
\hline Rwanda & 55 & 13 \\
\hline Senegal & 103 & 24 \\
\hline
\end{tabular}

\begin{tabular}{|c|c|c|}
\hline & $\$ \mathrm{mn}$ & $\$$ per person \\
\hline Seychelles & 8 & 133 \\
\hline Sierra Leone & 13 & 5 \\
\hline Somalia & 46 & 15 \\
\hline Sudan & 78 & 4 \\
\hline Swaziland & 15 & 32 \\
\hline Tanzania & 180 & 12 \\
\hline Togo & 34 & 16 \\
\hline Tunisia & 141 & 25 \\
\hline Uganda & 14 & 1 \\
\hline Upper Volta & 77 & 13 \\
\hline Zaire & 172 & 7 \\
\hline Zambia & 60 & 13 \\
\hline America $^{\mathrm{d}}$ & 1465 & 4 \\
\hline Argentina & 35 & 1 \\
\hline Bahamas & 1 & 3 \\
\hline Barbados & 4 & 18 \\
\hline Belize & 6 & 48 \\
\hline Bolivia & 49 & 9 \\
\hline Brazil & 148 & 1 \\
\hline Chile & 67 & 7 \\
\hline Colombia & 116 & 5 \\
\hline Costa Rica & 25 & 13 \\
\hline Cuba & 14 & 1 \\
\hline Dominican Republic & 26 & 6 \\
\hline Ecuador & 50 & 7 \\
\hline El Salvador & 29 & 7 \\
\hline French Guiana & 43 & 748 \\
\hline Guadeloupe & 112 & 320 \\
\hline Guatemala & 31 & 5 \\
\hline Guyana & 10 & 13 \\
\hline Haiti & 25 & 6 \\
\hline Honduras & 30 & 10 \\
\hline Jamaica & 23 & 12 \\
\hline Martinique & 124 & 347 \\
\hline Mexico & 55 & 1 \\
\hline Netherlands Antilles & 28 & 118 \\
\hline Nicaragua & 40 & 19 \\
\hline Panama & 28 & 17 \\
\hline Paraguay & 32 & 12 \\
\hline Peru & 83 & 5 \\
\hline St Pierre \& Miquelon & 10 & 1722 \\
\hline Surinam & 44 & 106 \\
\hline Trinidad \& Tobago & 5 & 5 \\
\hline Uruguay & 14 & 4 \\
\hline Venezuela & 15 & 1 \\
\hline Asia $^{\mathrm{d}}$ & 4790 & 4 \\
\hline Afghanistan & 45 & 2 \\
\hline Bangladesh & 610 & 8 \\
\hline Burma & 76 & 3 \\
\hline Cambodia & 179 & 23 \\
\hline India & 1097 & 2 \\
\hline Indonesia & 652 & 5 \\
\hline Iraq & 17 & 2 \\
\hline Israel & 257 & 76 \\
\hline Jordan & 103 & 39 \\
\hline Korea, South & 258 & 8 \\
\hline Laos & 58 & 18 \\
\hline Lebanon & 14 & 5 \\
\hline Malaysia & 70 & 6 \\
\hline Maldives & 1 & 10 \\
\hline Nepal & 37 & 3 \\
\hline Pakistan & 353 & 5 \\
\hline Philippines & 188 & 5 \\
\hline Singapore & 20 & 9 \\
\hline Sri Lanka & 92 & 7 \\
\hline Syria & 21 & 3 \\
\hline Thailand & 72 & 2 \\
\hline Yemen, North & 34 & 5 \\
\hline Yemen, South & 14 & 8 \\
\hline
\end{tabular}

\begin{tabular}{lrr}
\hline & $\$$ mn & $\$$ per person \\
Oceania $^{\text {d }}$ & 522 & 123 \\
Fiji & 16 & 29 \\
French Polynesia & 53 & 429 \\
Gilbert Islands & 6 & 90 \\
New Caledonia & 67 & 505 \\
New Hebrides & 12 & 129 \\
Pacific Islands, US & 74 & 650 \\
Papua New Guinea & 255 & 96 \\
Samoa, Western & 7 & 48 \\
Solomon Islands & 15 & 82 \\
Tonga & 2 & 25
\end{tabular}

Economic aid ${ }^{e}$ given by

communist developed countries

Average per year (1973-75)

\begin{tabular}{lcc}
\hline \multicolumn{3}{c}{ Outflow of economic aid by country } \\
& $\$$ mn $\%$ of gdp \\
Total & $\mathbf{2 8 2 8 *}$ & $\mathbf{0 . 2 8} * * *$ \\
Bulgaria & 59 & $0.39 * * *$ \\
China & 394 & $0.15 * * *$ \\
Czechoslovakia & 193 & $0.42 * * *$ \\
Germany, East & $115^{*}$ & $0.21 * * *$ \\
Hungary & 136 & $0.68 * * *$ \\
Poland & 136 & $0.18^{* * *}$ \\
Rumania & 418 & $1.55^{* * *}$ \\
Soviet Union & 1377 & $0.25 * * *$
\end{tabular}

Inflow of economic aid by region and country

\begin{tabular}{|c|c|c|}
\hline & $\$ \mathrm{mn}$ & $\$$ per person \\
\hline Africa ${ }^{\mathrm{d}}$ & 715 & 2 \\
\hline Algeria & 163 & 10 \\
\hline Cameroon & 25 & 4 \\
\hline Chad & 22 & 6 \\
\hline Egypt & 158 & 4 \\
\hline Ghana & 22 & 2 \\
\hline Guinea & 28 & 6 \\
\hline Mauritania & 13 & 10 \\
\hline Mozambique & 20 & 2 \\
\hline Niger & 18 & 4 \\
\hline Senegal & 17 & 4 \\
\hline Somalia & 21 & 7 \\
\hline Sudan & 45 & 3 \\
\hline Tanzania & 27 & 2 \\
\hline Tunisia & 18 & 3 \\
\hline Upper Volta & 37 & 6 \\
\hline Zaire & 38 & 2 \\
\hline Zambia & 20 & 4 \\
\hline America $^{\mathbf{d}}$ & 393 & 1 \\
\hline Argentina & 290 & 12 \\
\hline Brazil & 60 & 1 \\
\hline Chile & 7 & 1 \\
\hline Mexico & 22 & 0.4 \\
\hline Asia $^{\mathrm{d}}$ & 1423 & 1 \\
\hline Afghanistan & 235 & 12 \\
\hline Bangladesh & 94 & 1 \\
\hline Cambodia & 10 & 1 \\
\hline India & 177 & 0.3 \\
\hline Indonesia & 33 & 0.3 \\
\hline Iran & 169 & 5 \\
\hline Iraq & 141 & 13 \\
\hline Jordan & 58 & 22 \\
\hline Nepal & 27 & 2 \\
\hline Pakistan & 87 & 1 \\
\hline Sri Lanka & 32 & 2 \\
\hline Syria & 383 & 54 \\
\hline Europe & 289 & 1 \\
\hline Turkey & 289 & 7 \\
\hline
\end{tabular}

${ }^{a}$ Official development assistance, including both bilateral assistance and flows to and from multilateral institutions ${ }^{b} \mathrm{Gross}$ domestic product ${ }^{\mathrm{c}} \mathrm{This}$ total of inflows differs from the total of outflows mainly due to 'pipe-line effects' following from the difference in the time of inflows to multilateral institutions and the outflow from them to developing countries Including flows not shown separately 'Bilateral commitments of capital to developing countries Including Northern Marianas 